\title{
Impact of monopolar radiofrequency energy on subchondral bone viability
}

\author{
Peter Balcarek • Anke Kuhn • Arwed Weigel • \\ Tim A. Walde • Keno G. Ferlemann • \\ Klaus M. Stürmer · Karl-Heinz Frosch
}

Received: 26 April 2009/Accepted: 21 September 2009/Published online: 17 October 2009

(C) The Author(s) 2009. This article is published with open access at Springerlink.com

\begin{abstract}
The purpose of this study was to analyze the impact of monopolar radiofrequency energy treatment on subchondral bone viability. The femoral grooves of six chinchilla bastard rabbits were exposed bilaterally to monopolar radiofrequency energy for 2, 4 and $8 \mathrm{~s}$, creating a total of 36 defects. An intravital fluorescence bone-labeling technique characterized the process of subchondral bone mineralization within the 3 months following exposure to radiofrequency energy and was analyzed by widefield epifluorescence optical sectioning microscopy using an ApoTome. After $2 \mathrm{~s}$ of radiofrequency energy exposure, regular fluorescence staining of the subchondral bone was evident in all samples when compared to untreated areas. The depth of osteonecrosis after 4 and $8 \mathrm{~s}$ of radiofrequency energy treatment averaged 126 and $942 \mu \mathrm{m}$ at 22 days $(P<.05$; $P<.01)$. The $4 \mathrm{~s}$ treatment group showed no osteonecrosis after 44 days whereas the depth of osteonecrosis extended from $519 \mu \mathrm{m}$ at 44 days $(P<.01)$, to $281 \mu \mathrm{m}$ at 66 days $(P<.01)$ and to $133 \mu \mathrm{m}$ at 88 days $(P<.05)$ after $8 \mathrm{~s}$ of radiofrequency energy application. Though radiofrequency energy may induce transient osteonecrosis in the superficial zone of the subchondral bone, the results of this study suggest that post-arthroscopic osteonecrosis appears to be of
\end{abstract}

P. Balcarek $(\bowtie) \cdot$ A. Kuhn · T. A. Walde .

K. G. Ferlemann · K. M. Stürmer · K.-H. Frosch

Department of Trauma Surgery, Plastic-and Reconstructive Surgery, University Medicine, Georg-August University, Robert-Koch-Str. 40, 37075 Göttingen, Germany

e-mail: peter.balcarek@med.uni-goettingen.de

\footnotetext{
A. Weigel

Department of Neurophysiology and Cellular Biophysics, University Medicine, Georg-August University and Deutsche Forschungsgemeinschaft-Research Center for the Molecular Physiology of the Brain (FZT 103), Göttingen, Germany
}

only modest risk given the current clinical application in humans.

Keywords Osteonecrosis - Thermal chondroplasty · Radiofrequency energy $\cdot$ Monopolar

\section{Introduction}

In recent years, radiofrequency energy (RFE) devices have been widely used for arthroscopic thermal chondroplasty; however, their benefit has become more and more controversial. The treatment aims to contour and smooth fibrillated cartilage surface, decrease inflammatory response and prevent further collagen and proteoglycan loss through the preservation of chondrocyte viability $[1,2]$. Several investigations have revealed increasing evidence that RFE treatment may result in significant chondrocyte cell death, including partial involvement of the subchondral bone layer [3-11]. As a result, an association between RFE treatment and osteonecrosis has been hypothesized; however, the actual effect of RFE on subchondral bone viability remains to be elucidated $[4,5,12,13]$.

The dynamic process of bone formation, remodeling and regeneration in vivo can be examined by polychrome labeling using calcium-binding fluorochromes [14, 15]. Fluorochromes are only deposited at sites of active mineralization, thus they provide information regarding the temporal progression of bone remodeling processes and the direction of bone formation. To prove the hypothesis that monopolar RFE treatment might result in depletion of subchondral bone viability, an intravital fluorescence bonelabeling technique was used to investigate changes in bone mineralization within 3 months after RFE exposure in a rabbit in vivo model. 


\section{Materials and methods}

All animal experiments were performed with the approval of our institutional review board and the local government animal rights protection authorities. A total of twelve knees in six adult female rabbits (Chinchilla Bastard, body weight $>3 \mathrm{~kg}$ ) were used for this study. Animals were anesthetized with ketamine $(0.8 \mathrm{mg} / \mathrm{kg}$ body weight $)$ and xylazine ( $5 \mathrm{mg} / \mathrm{kg}$ body weight) intramuscularly. A medial parapatellar incision was made followed by lateral dislocation of the patella, which exposed the femoral groove. Using the Arthrex AR9600 ${ }^{\circledR}$ Orthopaedic Procedure Electrosurgical System and the Arthrex AR-9603-60 ablation probe (Bovie Medical Corporation St. Petersburg, Florida, USA), monopolar RFE was applied through continuous, stationary delivery for 2, 4 and $8 \mathrm{~s}$ on three different points of each femoral groove creating a total of 36 defects. The probe tip of the electrode was held stationary in freehand lightcontact mode to simulate operating room conditions. One surgeon (P. B.) performed all operations. The power setting was used as recommended by the manufacturer (Preset 7 , 40 Watt) and continuous, room temperate lavage solution (Ringer's lactate) was applied manually through a syringe during RFE application.

After RFE application layer-to-layer suturing of the medial retinaculum, and skin was performed using 4.0 Vicryl $\left(\right.$ Ethicon $^{\circledR}$, a Johnson \& Johnson Company, Langhorne, Pennsylvania, USA). Postoperatively, animals received an analgesic treatment with Carprofen $(5 \mathrm{mg} / \mathrm{kg}$ body weight) subcutaneously for 3 days. Within the following three-month period, all animals were housed in deep litter and showed species-appropriate behavior. None of the animals developed patellar luxation.

\section{Intravital fluorescence bone labeling}

After the operative procedure, all animals received a fluorescence agent (Merck, Darmstadt, Germany) every 22 days to label bone formation. Fluorescence agents are only deposited into vital bone at sites of active mineralization [14, 15]. Injections were administered subcutaneously according to the following schedule: Day 22xylenol-orange $(90 \mathrm{mg} / \mathrm{kg}$ body weight), Day 44 —calcein green $(10 \mathrm{mg} / \mathrm{kg}$ body weight), Day 66 -alizarin-complexone red (30 mg/kg body weight), Day 88 - tetracycline ( $25 \mathrm{mg} / \mathrm{kg}$ body weight). A three-month period was considered sufficient to investigate not only signs of osteonecrosis but also bone remodeling and regeneration in this animal model, using four different fluorochromes [16].

After 3 months, animals were euthanized, and the distal femora were harvested. Soft tissue and musculature were removed, and the femora were defatted in an alcohol series and embedded in methylmethacrylate. After solidification, $150( \pm 10) \mu \mathrm{m}$-thick sections were cut perpendicular to the longitudinal axis of the femur with a Leica SP 1600 innerhole saw-microtome (Leica, Bensheim, Germany). The saw blade had a thickness of $300 \mu \mathrm{m}$. The thickness of each slide was confirmed with a measuring device specifically for evaluating histological sections (Leica, Bensheim, Germany).

\section{High-resolution microradiography}

Microradiographs were performed on samples from the central section of each defect with KODAK Professional Industrex SR45 film (100NIF) and the Faxitron Microfocus Cabinet radiograph system (Hewlett-Packard, $50 \mu \mathrm{m}$ x-ray beam output; Model 43855A; Buffalo Grove, Illinois, USA). The bone sections were exposed to $40 \mathrm{kV}$ for $6 \mathrm{~min}$. The film was analyzed by light microscopy and a digital image processing system (Quantimet system, Leica DMRXE, Bensheim, Germany).

\section{Microscopy}

After the microradiographic procedure, sections were mounted on slides for microscopic analysis. Samples were analyzed and viewed on a Leica DM-IRBE microscope and on a Leica MZ-5 stereomacroscope (Leica Microsystems, Bensheim, Germany) for standard fluorescence microscopy. The optically sectioned fluorescence images were generated on an AxioObserver Z.1 microscope equipped with an ApoTome slider (Carl Zeiss, Inc, Germany). Settings were as follows: luminous source-EXFO X-Cite Series 120; filter sets-49, $38 \mathrm{HE}$ and 43; objective-EC Plan-NEOFLUAR 10x/0.3; scale- $0.65 \mu \mathrm{m} /$ pixel; grid-VL; camera-AxioCam MRm $1388 \times 1040$ pixel; software-AxioVision 4.6.3.0 (Carl Zeiss, Inc, Germany). The ApoTome principle uses a grid of stripes that is inserted into the microscope's field stop plane and thus projected onto the object. As the high spatial frequency of the grid rapidly attenuates with defocus, its modulation serves as a "focus label". The grid is shifted laterally in three defined steps relative to the sample. A camera takes a picture in each grid position. The three raw images are combined into a resultant optically sectioned image by on-line computation.

The depth of osteonecrosis was determined in the fluorescence preparations with a modified method according to Caffey et al. [3] (Fig. 1). Images were digitally acquired and analyzed with the help of the Leica QwinV3 System (Leica Microsystems, Bensheim, Germany). To measure the time dependence of osteonecrosis depth, a tangential line was drawn along the tidemark of the zone of calcified 


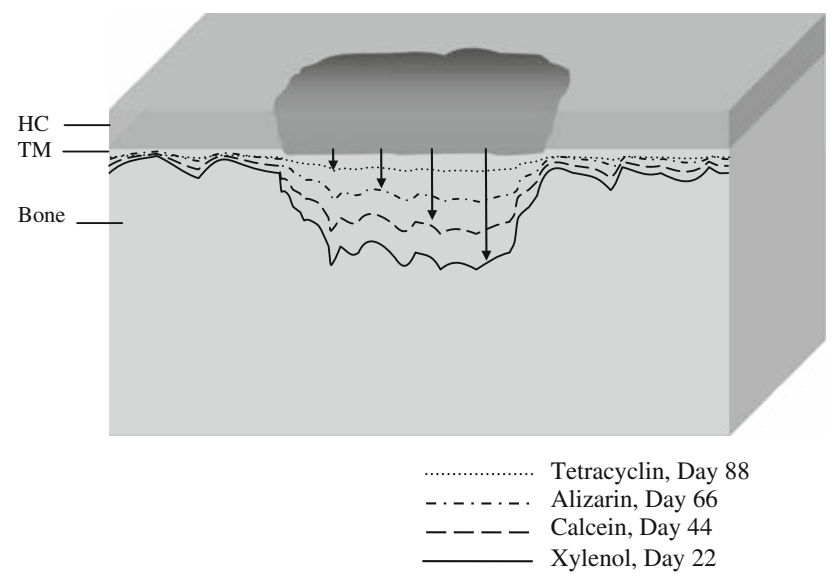

Fig. 1 Femoral groove cross-section to measure radiofrequency energy-induced thermal injury in a modified protocol according to Caffee et al. Arrowheads indicate time-dependent depth of thermal injury indicated by the demarcated half-circle of stained and unstained bone measured separately for xylenol, calcein, alizarin and tetrazyclin (HC Hyaline cartilage, TM Tidemark)

cartilage. From this line, a second line was drawn at an angle of $90^{\circ}$; the line extended to the depth of either xylenol, calcein, alizarin or tetracyclin fluorescence staining representing subchondral bone mineralization on day $22,44,66$ and 88, respectively. For each fluorochrome, six measurements were performed in the central aspect of each defect. Measurements were also performed in sham-operated areas of the femoral groove that were localized between RFE application points to provide a baseline of physiological bone mineralization beneath the tidemark in this animal model within a three-month period. The depth of osteonecrosis was defined as the difference between depths of fluorescence staining of the treatment groups and the sham group measured separately for each fluorochrome.

\section{Statistical analysis}

Statistical analyses were performed with GraphPad Prism ${ }^{\circledR}$ (Prism 4 Statistics Guide -Statistical analyses for laboratory and clinical researchers, GraphPad Software, Inc., San Diego, California, USA, 2003). Mean values and standard deviations are presented. The one-way ANOVA followed by Dunnett's Multiple Comparison post-test was used to compare the treatment groups with the sham group. A $P$ value less than .05 was considered statistically significant.

\section{Results}

A total of 36 defects were evaluated. Twelve defects represented each exposure time $(2,4$ or $8 \mathrm{~s}$ of monopolar

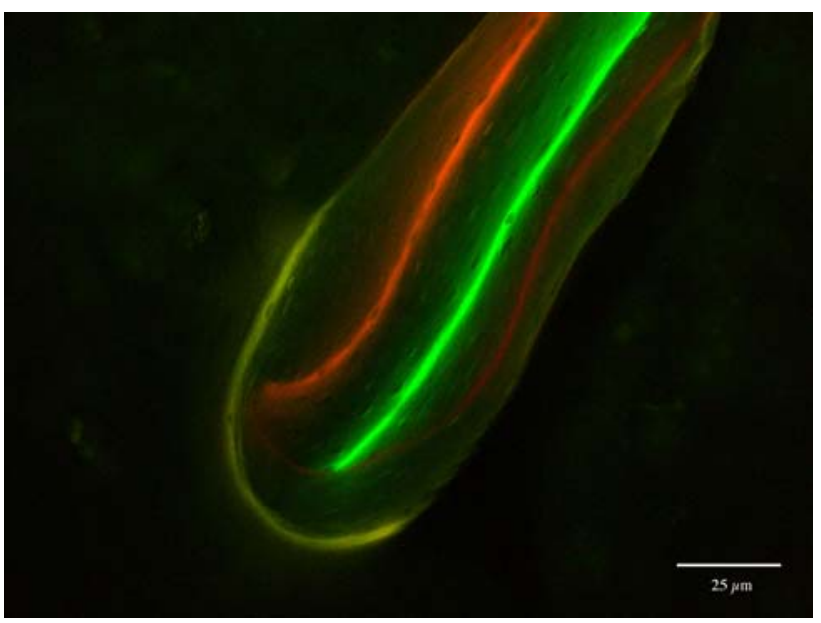

Fig. 2 Deposition of fluorescence agents: Day 22-xylenol orange, Day 44-calcein green, Day 66-alizarin-komplexon red, Day 88tetracycline yellow

RFE). Fluorescence microscopy confirmed standard administration of all fluorescence agents for every animal with the typical time-dependent fluorochrome deposition (Fig. 2).

Table 1 and Fig. 3 summarize the time course of subchondral bone mineralization of sham-operated and RFEtreated areas during the three-month period. In untreated areas, mean depth of subchondral bone mineralization beneath the tidemark was $145 \pm 117 \mu \mathrm{m}$ at 22 days, $143 \pm 73 \mu \mathrm{m}$ at 44 days, $134 \pm 63 \mu \mathrm{m}$ at 66 days and $110 \pm 59 \mu \mathrm{m}$ at 88 days.

After $2 \mathrm{~s}$ of RFE exposure, regular fluorescence staining of the subchondral bone was evident in all samples when compared to untreated areas (Fig. 4a, b). Mean depth of subchondral bone mineralization beneath the tidemark was $130 \pm 58 \mu \mathrm{m}$ at 22 days, $124 \pm 63 \mu \mathrm{m}$ at 44 days, $136 \pm 75 \mu \mathrm{m}$ at 66 days and $93 \pm 75 \mu \mathrm{m}$ at 88 days

Table 1 Summary of the time course of subchondral bone mineralization of sham-operated and RFE-treated areas during the threemonth period

\begin{tabular}{llrll}
\hline & Sham & \multicolumn{1}{l}{$2 \mathrm{~s}$} & \multicolumn{1}{l}{$4 \mathrm{~s}$} & $8 \mathrm{~s}$ \\
\hline $\begin{array}{l}\text { Xylenol (day } \\
\text { 22) }\end{array}$ & $145 \pm 117$ & $130 \pm 58$ & $271 \pm 174^{*}$ & $1087 \pm 201^{*}$ \\
Calcein (day 44) & $143 \pm 73$ & $124 \pm 63$ & $162 \pm 123$ & $662 \pm 207^{*}$ \\
$\begin{array}{l}\text { Alzarin (day 66) } \\
\text { Tetrazyclin (day }\end{array}$ & $110 \pm 63$ & $136 \pm 75$ & $150 \pm 84$ & $415 \pm 142^{*}$ \\
88 ) & & $93 \pm 75$ & $117 \pm 65$ & $243 \pm 90^{*}$ \\
\end{tabular}

Xylenol, calcein, alizarin and tetracyclin fluorescence staining represents subchondral bone mineralization beneath the tidemark on day 22, 44, 66 and 88 after RFE exposure. Values presented in $\mu \mathrm{m}$

$* P<.05$ (one-way ANOVA followed by Dunnett's Multiple Comparison post-Test) 


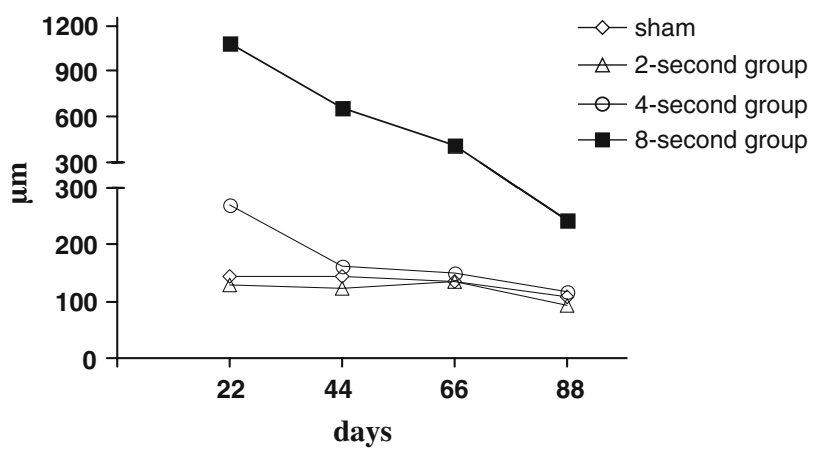

Fig. 3 Time course and depths of fluorescence labeling of vital subchondral bone beneath the tidemark over a three-month period in sham-operated and in RFE-treated areas after 2, 4 and $8 \mathrm{~s}$ of RFE application

showing no significant difference in comparison with untreated areas $(P>.05)$.

After $4 \mathrm{~s}$ of exposure, a significant recess of fluorescence labeling in the superficial zone of the subchondral bone layer was evident, extending to a mean maximum depth of $271 \pm 174 \mu \mathrm{m}$ at 22 days $(P<.01)$ (Fig. 4c). Depth of fluorescence labeling decreased to $162 \pm 123 \mu \mathrm{m}$ at 44 days $(P>.05)$ to $150 \pm 84 \mu \mathrm{m}$ at 66 days $(P>.05)$ and to $117 \pm 65 \mu \mathrm{m}$ at 88 days $(P>.05)$.
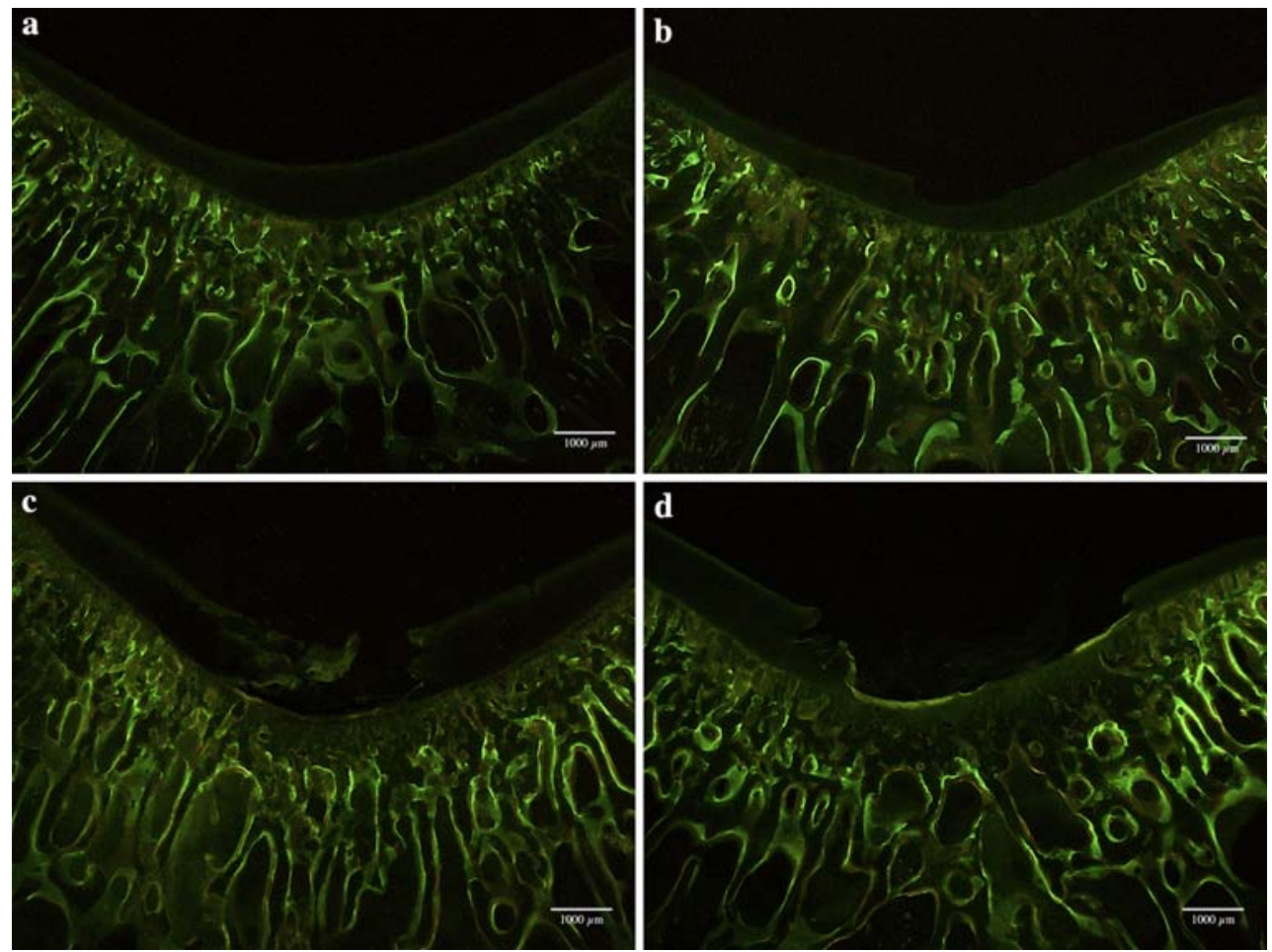

Fig. 4 a-d Each figure represents a cross-section through the femoral groove analyzed by fluorescence microscopy. a Shows an untreated area with regular deposition of the fluorescence agents and the weakly labeled intact articular cartilage. b Shows the central section of an area after $2 \mathrm{~s}$ of RFE exposure. The RFE probe created a halfthickness cartilage crater while subchondral bone mineralization

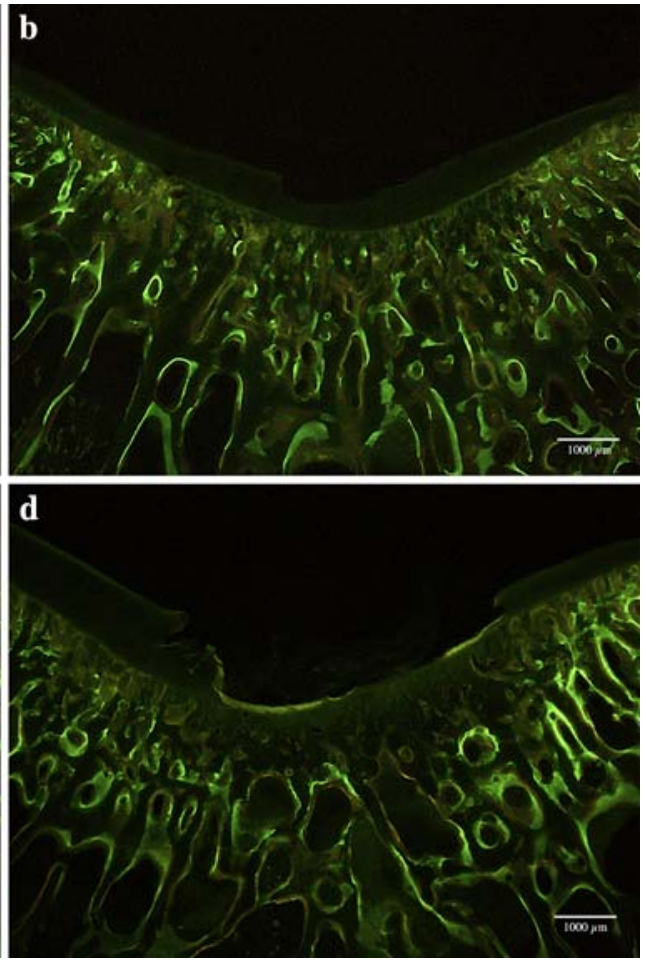

After $8 \mathrm{~s}$ of RFE application fluorescence labeling of vital bone extended to a mean maximum depth of $1087 \pm 201 \mu \mathrm{m}$ at 22 days $(P<.01)$, decreased to a mean depth of $662 \pm 207 \mu \mathrm{m}$ at 44 days $(P<.01)$ to $415 \pm 142 \mu \mathrm{m}$ at 66 days $(P<.01)$ and to $243 \pm 90 \mu \mathrm{m}$ at 88 days $(P<.01)$.

Our data show an average effective depth of osteonecrosis after 4 and $8 \mathrm{~s}$ of RFE treatment of 126 and $942 \mu \mathrm{m}$ at 22 days. The $4 \mathrm{~s}$ treatment group showed no osteonecrosis after 44 days whereas the depth of osteonecrosis decreased to $519 \mu \mathrm{m}$ at 44 days, to $281 \mu \mathrm{m}$ at 66 days and to $133 \mu \mathrm{m}$ at 88 days after $8 \mathrm{~s}$ of RFE application.

No structural differences in subchondral bone morphology were observed between treated and untreated samples when analyzed by microradiography, i.e., areas of osteonecrosis were not detectable with this method (Figs. 5a-d, 6).

\section{Discussion}

The most important finding of the present study was that depletion of subchondral bone viability might occur as a result of exposure to monopolar RFE. Several recent

remains regular. c Shows the central section of an area after $4 \mathrm{~s}$ of RFE exposure. A full-thickness cartilage defect is weakly labeled. In addition, a small recess of active bone mineralization is clear. d Shows an area after $8 \mathrm{~s}$ of RFE exposure. This sample yielded a well-demarcated half-circle border between the clearly stained vital and the unstained dead subchondral bone 

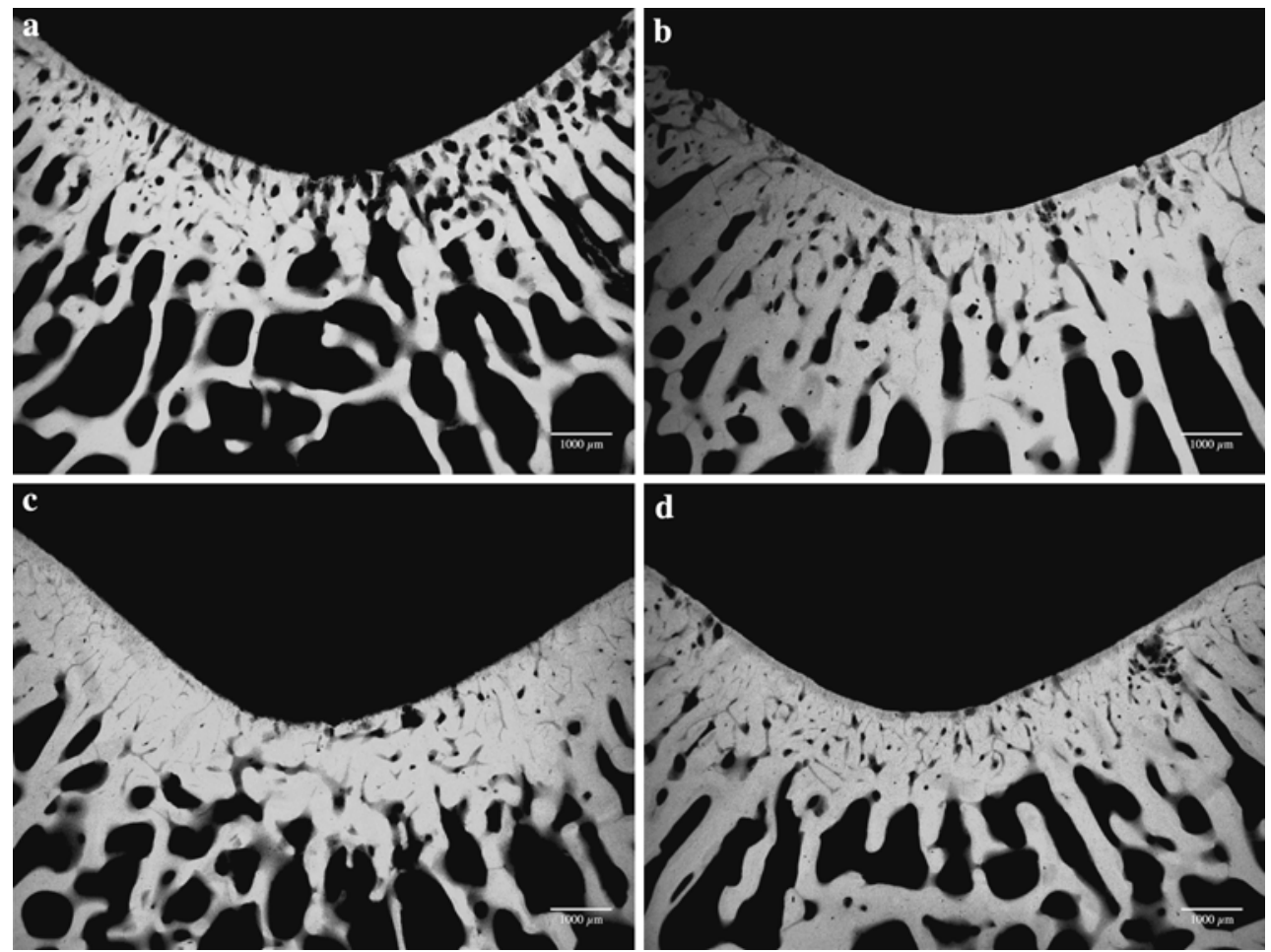

Fig. 5 a-d Each figure shows a cross-section through the femoral groove analyzed by microradiography. No difference in bone morphology was observed after $2 \mathrm{~s}(\mathbf{b}), 4 \mathrm{~s}$ (c) and $8 \mathrm{~s}$ (d) of RFE exposure in comparison with sham-operated bone (a)

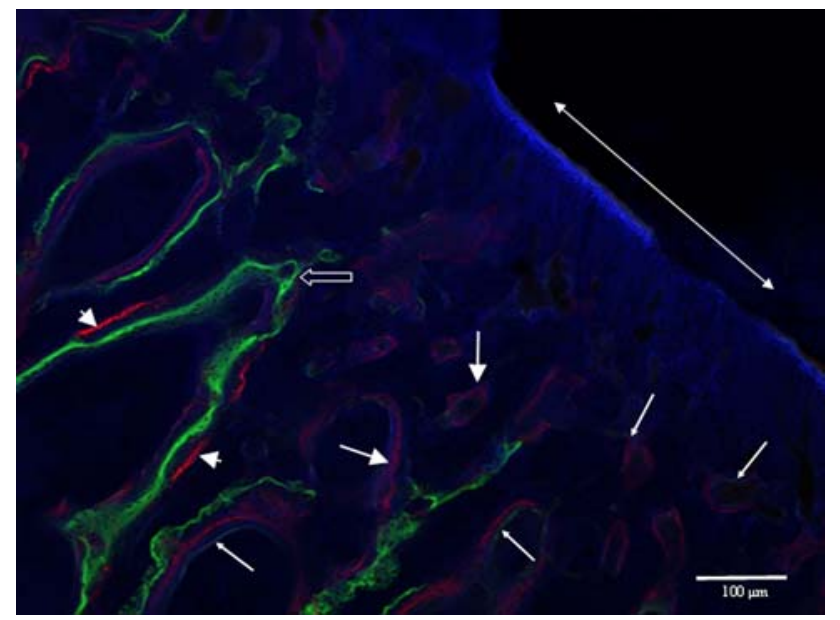

Fig. 6 ApoTome-generated fluorescence picture at the verge of an area after $8 \mathrm{~s}$ of RFE exposure. Double-headed arrow shows the treatment area with a full-thickness cartilage defect. Note the zone of osteonecrosis with the time-dependent remineralization. (Arrowhead Day 22; open arrow Day 44; large solid arrow Day 66; small solid arrow Day 88 (weakly pictured); blue autofluorescence)

studies have investigated the effect of RFE devices on articular cartilage $[1-6,8-11,17,18]$. Though thermal chondroplasty results in a smooth, contoured fibrillated cartilage surface and restored mechanical properties, detrimental consequences on chondrocyte viability have been reported with extended cartilage loss [6, 18]. Different experimental settings yield contradictory results with regard to the extent of chondrocyte cell death and damage to the underlying matrix. Visual examination by the surgeon is not sufficient to measure cellular damage. The clinical use of RFE is becoming more worrisome and many authors recommend a cautious approach toward the clinical use of RFE devices [5, 17].

Objective measurements of cell viability support the conclusion that RFE has the capability to cause significant chondrocyte cell death potentially extending to the level of the subchondral bone layer [3-5]. However, the actual effect of RFE on bone has not been investigated in detail, and avascular necrosis remains a major concern when thermally treating cartilage $[3,13]$. Therefore, this study was designed to determine the effect of RFE on subchondral bone viability using an intravital fluorescence bonelabeling technique. Our goal was not to determine the optimal conditions for performing thermal chondroplasty.

Secondary osteonecrosis of the knee has been previously described in case reports and small case series, both lacking comprehensive information on the pathophysiology. After conventional arthroscopy with partial meniscal resection an increased load transmitted to the already injured cartilage has been hypothesized to result in subchondral microfractures or changes in the subchondral blood supply, typically leading to avascular necrosis in the elderly [19, 20]. Although laser-assisted surgery-induced 
osteonecrosis as a result of thermal or photoacoustic tissue damage to the underlying matrix has been described, few reports have considered that a similar effect could result from the use of RFE devices [12, 13, 21, 22]. Most recently, Bonutti and colleagues presented a series of nineteen patients treated with knee arthroplasty for osteonecrosis. In this study, ten patients had undergone previous arthroscopy with associated RFE treatment. However, in their review of eleven reports, post arthroscopic osteonecrosis of the knee occurred most often after laser-assisted surgery or after an arthroscopic meniscectomy utilizing a standard basket punch. In only one report was osteonecrosis associated with the use of an RFE device. Therefore, the extent to which osteonecrotic lesions are due to applied energy from RFE devices, or to the often simultaneously performed meniscectomy is still under debate [13, 19, 20].

Heating of the cartilage surface with heat shock-induced chondrocyte cell death has been described in several reports after RFE treatment [6, 9-11]. The temperature at the surface and below the surface of the articular cartilage adjacent to the RFE probe reached $66-74^{\circ} \mathrm{C}$ upon stimulation with a monopolar device $[23,24]$. Such temperatures induce heat shock necrosis in osteoblasts [25]. Using the experimental settings of our study, we demonstrated that RFE has the potential to induce thermal injury to the superficial subchondral bone layer with increasing treatment time. Thus, this study supports the aforementioned hypothesis that monopolar RFE treatment might result in depletion of subchondral bone viability. Osteonecrosis averaged a maximum depth of $126 \mu \mathrm{m}$ after $4 \mathrm{~s}$ and $942 \mu \mathrm{m}$ after $8 \mathrm{~s}$ of exposure. Though recent clinical studies found no incidents of avascular necrosis 6 or 12 months after RFE treatment and, when compared to mechanical shaving, thermal chondroplasty resulted in greater smoothing of chondromalacic cartilage with decreased total tissue effects, it remains to be proven whether focal osteonecrotic lesions occur more frequently under clinical conditions than previously expected, becoming symptomatic in only in a small number of patients due to a stepwise regeneration [7, 26, 27].

The time course of bone regeneration in our study was markedly slowed in comparison with previous studies, supporting recent findings that heat-injured bone maintains only an inferior capacity to regenerate [16, 28]. Recent studies have shown that revascularization begins one to 2 weeks after radiofrequency thermal balloon angioplasty, but is not complete until 4 weeks following treatment [29]. Thus, the delayed progress of bone regeneration in our study may result from thermal injury not only to bone but also to vascular structures, depriving subchondral tissue of the blood supply required for rapid growth.

The intravital fluorescence bone-labeling technique allows investigation of the dynamic process of bone remodeling, representing the major advantage of this study. Nevertheless, several limitations deserve mention. First, during clinical use, RFE may be applied to cartilage in a "paint brush" manner-devices are not typically held stationary. Thus, according to Caffee et al. [3], we chose short treatment times to limit this effect. Second, our animal model did not support an arthroscopic procedure. The open surgery technique used in this study included the risk that the flowing irrigation fluid did not continuously surround the probe tip of the RFE device as is assured under arthroscopic settings. Thus, the temperature at the probe tip and the underlying tissue may have exceeded temperatures obtained under clinical conditions. In addition, we used room temperate lavage solution during RFE application though it has been shown that thermal chondroplasty with $37^{\circ} \mathrm{C}$ lavage solution resulted in less depth of chondrocyte death than with $22^{\circ} \mathrm{C}$ solution temperature [9]. However, room temperate lavage solution reflects more standard clinical settings. Third, cartilage thickness in our animal model was about one-fourth that of human cartilage; as cartilage tends to act as an insulator more energy may have been delivered to the subchondral bone than would occur in most circumstances under clinical applications in humans. However, Caffee and colleagues demonstrated that four out of five devices penetrated the full thickness of the cartilage to the bone with a treatment time of $3 \mathrm{~s}$ when applied to grade III cartilage defects in humans [3]. In addition, temperature requirements to achieve morphological change in articular cartilage are lower in already arthritic cartilage when compared to nonartritic cartilage [30]. These factors might influence the true effect of monopolar RFE when applied to arthritic articular cartilage and subsequently to subchondral bone under clinical settings. Fourth, we did not perform a power analysis. However, six measurements were performed in the central aspect of each defect. Twelve defects represented a 2, 4 or $8 \mathrm{~s}$ treatment time, respectively, resulting in 72 single measurements of each RFE exposure time. We do believe that this approach provided reliable data with respect to statistically significant differences. Finally, this study investigated the effect of only one RFE device. Different devices may exert varying effects on subchondral bone, depending on the energy setting, the probe tip design and the use of monopolar or bipolar energy in a non-contact or light-contact mode. However, the use of additional RFE devices was beyond the scope of this study.

Several recent studies have investigated the effect of RFE devices on articular cartilage and found detrimental consequences on chondrocyte viability with extended cartilage loss. In addition, data from our study indicate that the impact of monopolar RFE might result in depletion of subchondral bone viability with increasing treatment time. This finding is of clinical relevance as the field of RFE 
application is not limited to thermal chondroplasty. For example, RFE devices are used for notch preparation in anterior cruciate ligament reconstruction where RFE operates directly on bony tissue.

\section{Conclusions}

To the best of our knowledge, this is the first in vivo study to investigate possible RFE side effects on subchondral bone viability. With the described limitations and factors, which could not be added in the study design, the results of our study support the conclusion that increasing RFE treatment time may induce a focal, potentially transient osteonecrosis in the superficial zone of the subchondral bone. Under these conditions, the critical application time of monopolar RFE with regard to osteonecrosis was about $4 \mathrm{~s}$ of stationary delivery. Given the results of our study, with only a limited extent of osteonecrosis as a result of a relatively long stationary delivery of RFE, we would conclude that the complication of postarthroscopic osteonecrosis appears to be only a modest risk with the current clinical application mode (paint-brush pattern, short treatment time) in humans.

Acknowledgments The authors express their thanks to R. Castro and $\mathrm{F}$. Kauer for excellent experimental assistance.

Open Access This article is distributed under the terms of the Creative Commons Attribution Noncommercial License which permits any noncommercial use, distribution, and reproduction in any medium, provided the original author(s) and source are credited.

\section{References}

1. Kaplan L, Uribe JW (2009) The acute effects of radiofrequency energy in articular cartilage: an in vitro study. Arthroscopy $16: 2-5$

2. Turner AS, Tippett JW, Powers BE, Dewell RD, Mallinckrodt CH (1998) Radiofrequency (electrosurgical) ablation of articular cartilage: a study in sheep. Arthroscopy 14:585-591

3. Caffey S, McPherson E, Moore B, Hedman T, Vangsness CT Jr (2005) Effects of radiofrequency energy on human articular cartilage: an analysis of 5 systems. Am J Sports Med 33:10351039

4. Edwards RB 3rd, Hayashi K, Lu Y, Markel MD (2001) The acute effects of radiofrequency energy in articular cartilage: an in vitro study. Arthroscopy 17:112-113

5. Edwards RB 3rd, Lu Y, Nho S, Cole BJ, Markel MD (2002) Thermal chondroplasty of chondromalacic human cartilage. An ex vivo comparison of bipolar and monopolar radiofrequency devices. Am J Sports Med 30:90-97

6. Kääb MJ, Bail HJ, Rotter A, Mainil-Varlet P, apGwynn I, Weiler A (2005) Monopolar radiofrequency treatment of partial-thickness cartilage defects in the sheep knee joint leads to extended cartilage injury. Am J Sports Med 33:1472-1478
7. Lotto ML, Wright EJ, Appleby D, Zelicof SB, Lemos MJ, Lubowitz JH (2008) Ex vivo comparison of mechanical versus thermal chondroplasty: assessment of tissue effect at the surgical endpoint. Arthroscopy 24:410-415

8. Lu Y, Edwards RB 3rd, Cole BJ, Markel MD (2001) Thermal chondroplasty with radiofrequency energy. An in vitro comparison of bipolar and monopolar radiofrequency devices. Am J Sports Med 29:42-49

9. Lu Y, Edwards RB 3rd, Nho S, Cole BJ, Markel MD (2002) Lavage solution temperature influences depth of chondrocyte death and surface contouring during thermal chondroplasty with temperature-controlled monopolar radiofrequency energy. Am J Sports Med 30:667-673

10. Lu Y, Edwards RB 3rd, Nho S, Heiner JP, Cole BJ, Markel MD (2002) Thermal chondroplasty with bipolar and monopolar radiofrequency energy: effect of treatment time on chondrocyte death and surface contouring. Arthroscopy 18:779-788

11. Lu Y, Hayashi K, Hecht P, Fanton GS, Thabit G 3rd, Cooley AJ, Edwards RB, Markel MD (2000) The effect of monopolar radiofrequency energy on partial-thickness defects of articular cartilage. Arthroscopy 16:527-536

12. Bonutti PM, Seyler TM, Delanois RE, McMahon M, McCarthy JC, Mont MA (2006) Osteonecrosis of the knee after laser or radiofrequency-assisted arthroscopy: treatment with minimally invasive knee arthroplasty. J Bone Joint Surg Am 88(Suppl. 3): 69-75

13. Encalada I, Richmond JC (2004) Osteonecrosis after arthroscopic meniscectomy using radiofrequency. Arthroscopy 20:632-636

14. Frost HM (1969) Tetracycline-based histological analysis of bone remodeling. Calcif Tissue Res 3:211-237

15. Rahn BA, Perren SM (1979) Calcein blue as a fluorescent label in bone. Experentia 26:519

16. Frosch K-H, Gröll C, Viereck V, Drengk A, Stürmer KM, Dresing K (2005) Cell coated fibrin glue- and polymer fleece-cell constructs do not induce orthopic bone formation in the rabbit. Osteologie 14:162-171

17. Ryan A, Bertone AL, Kaeding CC, Backstrom KC, Weisbrode SE (2003) The effects of radiofrequency energy treatment on chondrocytes and matrix of fibrillated articular cartilage. Am J Sports Med 31:386-391

18. Uthamanthil RK, Edwards RB, Lu Y, Manley PA, Athanasiou KA, Markel MD (2006) In vivo study on the short-term effect of radiofrequency energy on chondromalacic patellar cartilage and its correlation with calcified cartilage pathology in an equine model. J Orthop Res 24:716-724

19. Muscolo DL, Costa-Paz M, Makino A, Ayerza MA (1996) Osteonecrosis of the knee following arthroscopic meniscectomy in patients over 50-years old. Arthroscopy 12:273-279

20. Pruès-Latour V, Bonvin JC, Fritschy D (1998) Nine cases of osteonecrosis in elderly patients following arthroscopic meniscectomy. Knee Surg Sports Traumatol Arthrosc 6:142-147

21. Janzen DL, Kosarek FJ, Helms CA, Cannon WD Jr, Wright JC (1997) Osteonecrosis after contact neodymium:yttrium aluminum garnet arthroscopic laser meniscectomy. AJR Am J Roentgenol 169:855-858

22. Möller KO, Lind BM, Karcher K, Hohlbach G (1994) Holmium laser versus mechanical cartilage resection. Comparative studies in the rabbit arthrosis model. Langenbecks Arch Chir 379:84-94

23. Kaplan LD, Ernsthausen JM, Bradley JP, Fu FH, Farkas DL (2003) The thermal field of radiofrequency probes at chondroplasty settings. Arthroscopy 19:632-640

24. Shellock FG (2001) Radiofrequency energy induced heating of bovine articular cartilage: comparison between temperaturecontrolled, monopolar, and bipolar systems. Knee Surg Sports Traumatol Arthrosc 9:392-397 
25. Li S, Chien S, Brånemark PI (1999) Heat shock-induced necrosis and apoptosis in osteoblasts. J Orthop Res 17:891-899

26. Barber FA, Iwasko NG (2006) Treatment of grade III femoral chondral lesions: mechanical chondroplasty versus monopolar radiofrequency probe. Arthroscopy 22:1312-1317

27. Cetik O, Cift H, Comert B, Cirpar M (2009) Risk of osteonecrosis of the femoral condyle after arthroscopic chondroplasty using radiofrequency: a prospective clinical series. Knee Surg Sports Traumatol Arthrosc 17:24-29

28. Stürmer KM, Kehr H, Schmit-Neuerburg KP (1979) Does lowfrequency magnetic field and alternating voltage improve bone healing? Zbl Chirurgie 104:777 (German)
29. Kaplan J, Barry KJ, Connolly RJ, Nardella PC, Hayes LL, Lee BI, Waller BF, Becker GJ, Callow AD (1939) Healing after arterial dilatation with radiofrequency thermal and nonthermal balloon angioplasty systems. J Invest Surg 6:33-52

30. Kaplan LD, Ionescu D, Ernsthausen JM, Bradley JP, Fu FH, Farkas DL (2004) Temperature requirements for altering the morphology of osteoarthritic and nonarthritic articular cartilage: in vitro thermal alteration of articular cartilage. Am J Sports Med 32:688-692 